\title{
Patientensicherheit in der psychiatrischen Versorgung
}

\author{
Anna Mascherek \\ Dr., Wissenschaftliche Mitarbeiterin Stiftung Patientensicherheit Schweiz
}

Im Rahmen des einjährigen Projekts «Patientensicherheit in der psychiatrischen Versorgung» möchte Patientensicherheit Schweiz gemeinsam mit Expert(inn)en aus der Schweiz das Thema praxisbezogen, interdisziplinär und sektorenübergreifend aufarbeiten. Ziel ist es, einen Aktionsvorschlag mit Themen, Projekten und Aktivitäten zur Verbesserung der Patientensicherheit in der psychiatrischen Versorgung in der Schweiz vorzulegen, der nach Dringlichkeit priorisiert ist.

Die Patientensicherheit nimmt vor allem in der somatischen Akutmedizin einen zunehmend grösseren Stellenwert ein. Obwohl Aspekte der Patientensicherheit in der somatischen Medizin auch für die psychiatrische Versorgung gelten, sind andere Aspekte sehr spezifisch. Die Besonderheiten sind einerseits in der Patientengruppe und dem Wesen der Erkrankungen zu finden, andererseits ergeben sich Anliegen der Patientensicherheit durch das Setting, in dem die Behandlung stattfindet, und durch die Behandlung selber [1]. Dennoch ist die systematische Auseinandersetzung mit Patientensicherheit in der psychiatrischen Versorgung in der Schweiz heute noch unterentwickelt. Gleichzeitig wird die psychiatrische Versorgung immer wichtiger, wenn man Prävalenz und Krankheitslast berücksichtigt. In einem Bericht des Schweizerischen Gesundheitsobservatoriums zur psychischen Gesundheit in der Schweiz heisst es, dass pro Jahr knapp 40\% der erweiterten EUBevölkerung (inkl. Schweiz) an einer psychischen Störung erkranken. Obwohl die Behandlungsmöglichkeiten relativ gut sind, zeigt der Bericht auch, dass rund zwei Drittel dieser psychischen Störungen unbehandelt bleiben [2, 3]. Zusätzlich stellen die Folgen psychischer Erkrankungen eine ökonomische Last dar. Zwar ist die Zahl der IV-Neuberentungen seit 2003 insgesamt rückläufig, der Anteil aufgrund psychischer Krankheiten jedoch gestiegen. Im Jahr 2013 waren von 230000 Berentungen in der Schweiz fast die Hälfte auf psychische Erkrankungen zurückzuführen [4]. Die Unterschiede zur somatischen Medizin und die vorhandene $\mathrm{Zu}$ nahme notwendiger psychiatrischer Behandlungen erfordern eine systematische Auseinandersetzung mit dem Thema. Vor allem auch, weil Risiken für die Patientensicherheit, die spezifisch für die psychiatrische Versorgung sind, schwerwiegende Themenbereiche um- fassen. Hierzu gehören beispielsweise Suizid, Fehldiagnosen sowie Zwangsmassnahmen (vgl. [5]). Im englischen Gesundheitssystem wird Suizid im Rahmen der psychiatrischen Behandlung bereits als sogenannter "never event» klassifiziert [6]. "Never events» sind definiert als Ereignisse, die eindeutig identifizier- und messbar sind, schwerwiegend sind (bspw. zu Tod oder substantieller Beeinträchtigung führen) und grundsätzlich vermeidbar wären [7]. Das US-amerikanische National Quality Forum führt Suizid, versuchten Suizid und Selbstverletzung mit schwerer Schädigung als meldepflichtiges schweres Patientensicherheitsereignis [8]. Suizidprävention muss also ein zentrales Anliegen der Patientensicherheit sein. Weiterhin stellt sich auch die Frage nach strukturellen und institutionellen $\mathrm{Pa}$ tientensicherheitsrisiken. Beispielsweise sind Auswirkungen von Stigmatisierung auf die konkreten Behandlungsangebote eine wichtige Frage auf strategischer und politischer Ebene [5]. In einer aktuellen Untersuchung aus der Schweiz [9] werden zentrale Risiken und Themen des klinischen Risikomanagements in der Schweiz im Mental-Health-Bereich erfasst und dargestellt. Auch für die Schweiz gelten nach dieser Studie die internationalen Hotspots. Behandlungsfehler, Selbst- und Fremdgefährdung durch aggressives Verhalten und spezifische Risiken psychiatrischer Patienten werden als drängende Themen benannt. Auch wenn die Studie einen wichtigen Beitrag leistet, um die Aufmerksamkeit für das Thema zu erhöhen, besteht die Notwendigkeit, auch andere Versorgungseinrichtungen und Expert(inn)en aus weiteren Bereichen als dem klinischen Risikomanagement in die Diskussion miteinzubeziehen. Obwohl die wissenschaftliche Literatur zu diesem Thema sehr begrenzt ist, werden einige Aspekte der Patientensicherheit immer wieder diskutiert, ak- 
tuell beispielsweise im Kontext der Diskussion um Zwangsmassnahmen oder Patientenverfügungen. Im Schweizer Manifest für Public Mental Health wird das Thema Patientensicherheit nur indirekt thematisiert, indem die dramatischen Folgen von verspäteten Behandlungen benannt werden (bspw. Suizid und Zwangsmassnahmen) [10]. Die indirekte Thematisierung ist symptomatisch für das Themenfeld: Oft werden Einzelaspekte herausgegriffen und prominent dargestellt, während ein gesamthafter thematischer Rahmen fehlt. Zusätzlich gehen Bemühungen oft in allgemeinen Qualitätsverbesserungsprojekten auf, in denen Patientensicherheit keinen eigenständigen Schwerpunkt bildet.

\section{Ziele und Fragestellungen}

Vor diesem Hintergrund hat Patientensicherheit Schweiz das Projekt «Patientensicherheit in der psychiatrischen Versorgung» lanciert, welches das Thema für die Schweiz praxisbezogen, interdisziplinär und sektorenübergreifend aufarbeiten soll. Die Sensibilisierung für dieses Thema soll erhöht und der dringlichste Handlungsbedarf aufgezeigt werden. Das Projekt wird massgeblich von der FMH finanziert und hat eine geplante Laufzeit von einem Jahr (bis Ende 2015).

\section{Erste Erkenntnisse}

In einem ersten Projektteil wurde eine RoundtableDiskussion mit Expert(inn)en aus der psychiatrischen Versorgung lanciert. Personen aus dem ambulanten und stationären Setting, aus dem Kinder- und Jugendsowie Erwachsenenbereich, verschiedenen klinischen Schwerpunkten und Vertreter unterschiedlicher Berufsgruppen und Patientenvertreter waren eingeladen, um das Thema aus den verschiedenen Perspektiven einzugrenzen. Das grosse Engagement der beteiligten Fachpersonen zeigt, dass das Thema Patientensicherheit auch «in der Realität» als wichtig wahrgenommen wird und kein «theoretisches Problemfeld» ist.

Eine erste Auswertung des Roundtables zeigt, dass die $\mathrm{Ab}$ - und Eingrenzung des Themas schwierig ist. Die präzise Abgrenzung von Patientensicherheit und Qualität stellt eine noch grössere Herausforderung dar als in der somatischen Medizin. Oft ungeklärte Fragen sind beispielsweise «Was sind Fehler in der psychiatrischen Versorgung? Wie kann man Fehler in der psychiatrischen Versorgung messen? Muss ein Fehler immer 'sichtbar' sein? Was sind zum Beispiel Nebenwirkungen von und Schädigungen durch Therapien?» Diese Aspekte sind in der psychiatrischen Versorgung deutlich schwieriger zu beantworten als in der somatischen.

Weiterhin zeigen die Ergebnisse des Roundtables, dass spezifische Merkmale der Patientenkollektive beson- dere Herausforderungen für die Gewährleistung der Patientensicherheit sein können. Die psychiatrische Erkrankung an sich beeinträchtigt den Patienten unter Umständen in seiner Person als Ganzes. Die Fragen nach der Autonomie des Patienten oder nach Selbstund Fremdgefährdung sind untrennbar mit der Person und der Krankheit verbunden. Unter Umständen können die Punkte aber mit dem Patienten selbst nicht diskutiert werden, da er durch die Erkrankung in der Urteilsfähigkeit in Bezug auf genau diese Fragen beeinträchtigt ist. Die Trennung zwischen «Erkrankung» und «Person» kann auch Aspekte der Patientensicherheit betreffen, wenn es beispielsweise um die Frage der Behandlung vs. Nichtbehandlung, um das Einleiten von Zwangsmassnahmen oder um die Diagnosestellung geht. Dass die Beurteilung einer Person als Ganzes, in ihrer Integrität, ihrem Verhalten, Empfinden und Erleben, und allenfalls entsprechende Massnahmen, die in diese Bereiche eingreifen, Teil der psychiatrischen Behandlung darstellen, eröffnet also per definitionem einen sensiblen Bereich der Patientensicherheit. Wenn hinzukommt, dass eine Person aufgrund der Schwere ihrer Beeinträchtigung nicht für sich selbst Verantwortung übernehmen kann, und möglicherweise auch die Kommunikation mit dem Behandlungsteam nicht oder nur schwer möglich ist, ist das Risiko für Fehler und Fehleinschätzungen zum Schaden des Patienten besonders gross. Diese Besonderheit ist also sicher eine grosse Herausforderung für die Patientensicherheit in der psychiatrischen Versorgung. Eine weitere Besonderheit in Abgrenzung zur somatischen Medizin ist die direkte Wechselwirkung, in der die psychiatrisch Betroffenen mit ihrer Umgebung / dem Kontext, in dem sie sich befinden, stehen. Hieraus ergibt sich eine sehr individuelle, besondere Vulnerabilität. Symptome einer Erkrankung sind nicht alle absolut, sondern stellen sich zum Teil abhängig von der sozialen und physikalischen Umgebung der betroffenen Person individuell dar. Diese gewisse Variabilität gilt es zu erkennen und in die Behandlung miteinzubeziehen. Diese Variabilität und die damit einhergehenden Herausforderungen finden sich auch in Aspekten der Patientensicherheit wieder. In einem zweiten Teil des Roundtables wurden Hotspots diskutiert, also Risiko-Felder, die besonders schwerwiegend und zentral für die Patientensicherheit sind. Die Ergebnisse zeigen, dass es in einigen Bereichen klare Überschneidungen mit der somatischen Medizin gibt, andere Aspekte aber spezifisch sind. Auch in der psychiatrischen Versorgung sind die Medikationssicherheit und die Problematik von Schnittstellen aller Art wesentliche Hotspots, wenn auch innerhalb der Themengebiete unterschiedliche Schwerpunkte auftauchen. Für die Medikationssicherheit ergeben sich Risiken 
durch Wechselwirkungen und Nebenwirkungen von Psychopharmaka und somatischen Medikamenten. Hinzu kommt das Monitoring und Follow-up von somatischen Symptomen im Rahmen der psychiatrischen Versorgung und umgekehrt.

Besonders für die psychiatrische Versorgung sind sicher die zentralen Themen Suizid, Aggression (Selbst- und Fremdgefährdung) und Zwangsmassnahmen. Obwohl in diesen Bereichen bereits viele Anstrengungen unternommen wurden, bleiben sie «Dauerbrenner». Vor allem, weil sie, wenn Fehler passieren, dramatisch und schwerwiegend sein können. Weitere Hotspots sind die Fragen nach Unter-, Über- und Fehldiagnostik und daraus entstehende Risiken für die Patientensicherheit sowie das Thema der Stigmatisierung. Auch wenn Stigmatisierung auf den ersten Blick ein gesellschaftliches Thema ist, hat es in der Konsequenz deutlichen Impact auf die Patientensicherheit. So werden beispielsweise aus Angst vor Stigmatisierung Behandlungsmöglichkeiten nicht aufgesucht. Ebenso besteht die Gefahr, dass psychiatrische Patienten mit somatischen Beschwerden zu wenig ernst genommen werden, als dass es zu einer adäquaten Abklärung dieser Beschwerden käme, oder dass Patienten auf somatischen Abteilungen mit ihren psychiatrischen Beschwerden nicht ausreichend wahrgenommen werden. Hier besteht Bedarf am Ausbau und Inanspruchnahme einer besseren Vernetzung und Zusammenarbeit der verschiedenen medizinischen Disziplinen.

\section{Bereits bestehende Aktivitäten}

In einem zweiten Projektteil wurden vorhandene Aktivitäten im Rahmen einer Umfrage systematisch und umfassend erfasst. Es bestehen bereits viele lokale Projekte und Aktivitäten, um die Patientensicherheit in der psychiatrischen Versorgung zu erfassen und/oder zu verbessern. Es handelt sich oftmals um Projekte im Bereich des Aggressionsmanagements, der Suizidprävention oder dem Umgang mit Zwangsmassnahmen. Auch im Rahmen der Umfrage zeigt sich, dass eine besondere Schwierigkeit zwischen der Abgrenzung von Patientensicherheit und Qualität besteht.

Nach Abschluss der ersten beiden Projektteile ergeben sich folgende wesentliche Erkenntnisse:

- Das Engagement der Fachpersonen unterstreicht, dass das Thema Patientensicherheit in der psychiatrischen Versorgung wichtig und drängend ist. Aktivitäten und Massnahmen werden auch von Personen im Feld eindeutig begrüsst.

- Es bestehen vielfältige Hotspots. Zum Teil finden sich Überschneidungen zur Somatik, viele Aspekte sind jedoch spezifisch für die psychiatrische Versorgung.
- Als eine nationale Netzwerkorganisation ist Patientensicherheit Schweiz geeignet, die vielfältigen, bestehenden Einzelaktionen zu bündeln und zu systematisieren. Dies ermöglicht den sparsamen Einsatz von Ressourcen bei gleichzeitig grösstem Nutzen für die Patienten. Zusätzlich gewinnt durch nationale Aktivitäten das Thema an Wichtigkeit; es kann sensibilisiert werden und nicht zuletzt einer Patientengruppe zu einer Lobby verholfen werden, die sich für eine Verbesserung der Patientensicherheit in der psychiatrischen Versorgung stark macht.

\section{Ausblick}

Patientensicherheit Schweiz wird in einem abschliessenden Teil des Projekts in Zusammenarbeit mit Fachexpert(inn)en die gewonnenen Informationen zusammentragen, filtern und ausdifferenzieren. Auf der Basis einer fundierten Definition und Eingrenzung des Themas sowie unter Berücksichtigung der bereits vorhandenen Aktivitäten soll ein Handlungsplan für die Bereiche Forschung, Praxis sowie Aus- und Weiterbildung erstellt werden, der die grössten Wissens- und Handlungsdefizite aufzeigt. Die Vorschläge werden durch Expert(inn)en validiert, konkretisiert und priorisiert, um den Handlungsbedarf breit abstützen und aufzeigen zu können. Ziel ist es, einen Aktionsvorschlag mit Themen, Projekten und Aktivitäten zur Verbesserung der Patientensicherheit in der psychiatrischen Versorgung in der Schweiz zu erstellen, der nach Dringlichkeit priorisiert ist. Das Ergebnis des Projekts soll als Grundlage dienen, um das Thema in Zukunft gut strukturiert, national vernetzt und mit einem sorgsamen Blick auf die Betroffenen anzugehen.

Literatur

1 Brickell TA, Nicholls TL, Procyshyn RM, McLean C, Dempster RJ, Lavoie JAA, et al. Patient safety in mental health. Edmonton, Alberta: Canadian Patient Safety Institute and Ontario Hospital Association; 2009.

2 Schuler D, Burla L. La santé psychisque en Suisse. Monitorage 2012 (Obsan rapport 52). Neuchâtel: Observatoire suisse de la santé; 2012.

3 Organisation mondiale de la santé. Santé mentale: relever les défis, trouver des solutions. Danemark: OMS; 2006.

4 Office fédéral des assurances sociales. Statistiques de la sécurité sociale. Statistique AI 2013. Berne; 2014.

5 Brickell TA, McLean C. Emerging issues and challenges for improving patient safety in mental health: A qualitative analysis of expert perspectives. Journal of Patient Safety. 2011;7(1):39-44.

6 NHS England. The never events list; 2013/14 update. 2013 [cited 2015 Jun 4]. Available from: www.england.nhs.uk/wp-content/ uploads/2013/12/nev-ev-list-1314-clar.pdf

7 Austin JM, Pronovost PJ. «Never Events» and the Quest to Reduce Preventable Harm. Jt Comm J Quality Safety. 201541(6):279-88.

8 The National Quality Forum. List of SREs. 2015 [cited 2015 Jun 4]. www.qualityforum.org/Topics/SREs/List_of_SREs.aspx\#sre3

9 Briner M, Manser T. Clinical risk management in mental health: a qualitative study of main risks and related organizational management practices. BMC Health Serv Res. 2013;13(1):44.

10 Santé publique Suisse. Manifeste suisse relatif à la Public Mental Health. Merenschwand: Edubook; 2014. 